\title{
Absence of p300 induces cellular phenotypic changes characteristic of epithelial to mesenchyme transition
}

\author{
D Krubasik', NG lyer², WR English', AA Ahmed², M Vias², C Roskelley ${ }^{3}$, JD Brenton ${ }^{2}$, C Caldas² and \\ G Murphy,
}

'Department of Oncology, University of Cambridge, Cambridge Institute for Medical Research, Addenbrooke's Hospital, Hills Road, Cambridge CB2 2XY, UK: ${ }^{2}$ Cancer Genomics Program, Department of Oncology, University of Cambridge, Hutchison/MRC Research Centre, Hills Road, Cambridge CB2 2XZ, UK; ${ }^{3}$ Department of Anatomy, University of British Columbia, 2177 Westbrook Mall, Vancouver BC V66T IZ3, UK

p300 is a transcriptional cofactor and prototype histone acetyltransferase involved in regulating multiple cellular processes. We generated $\mathrm{p} 300$ deficient $\left(\mathrm{p} 300^{-}\right.$) cells from the colon carcinoma cell line HCTI I 6 by gene targeting. Comparison of epithelial and mesenchymal proteins in P300- with parental HCTII 6 cells showed that a number of genes involved in cell and extracellular matrix interactions, typical of 'epithelial to mesenchyme transition' were differentially regulated at both the RNA and protein level. p300 cells were found to have aggressive 'cancer' phenotypes, with loss of cell-cell adhesion, defects in cell-matrix adhesion and increased migration through collagen and matrigel. Although migration was shown to be metalloproteinase mediated, these cells actually showed a downregulation or no change in the level of key metalloproteinases, indicating that changes in cellular adhesion properties can be critical for cellular mobility.

British Journal of Cancer (2006) 94, I326- 1332. doi: I0.1038/sj.bjc.6603 I 0 I www.bjcancer.com

Published online 18 April 2006

(c) 2006 Cancer Research UK

Keywords: p300; HCTI I6; homologous recombination; E-cadherin

p300 was initially cloned as an adenoviral E1A-binding protein and subsequently characterised as a transcriptional coactivator with histone acetyl transferase activity. It promotes gene transcription by bridging DNA-binding transcription factors and the basal transcription machinery, by providing a scaffold to integrate transcription factors and by modifying the activity of transcription factors and chromatin through direct acetylation of specific lysine residues. p300 and its close homologue CREB-binding protein (CBP) play a key role in a diverse array of cellular processes including cell cycle regulation, proliferation, differentiation, apoptosis, DNA damage repair and adhesion, as well as embryonic development (Chan and La Thangue, 2001; Iyer et al, 2004b). Mutations in p300/CBP have been found in a number of human cancers. Bi-allelic somatic mutations in the p300 gene have been identified in gastric, colon and breast cancers (primary tumours and cancer cell lines), and some of these mutations clearly result in inactivated or truncated protein products (Muraoka et al, 1996; Gayther et al, 2000; Ozdag et al, 2002). While this gave rise to the view that $\mathrm{p} 300$ could function as a classical tumour suppressor, it is still unclear how a loss of p300 and CBP could contribute to tumorigenesis (Goodman and Smolik, 2000).

It has been recently proposed that a major process contributing to tumour progression is epithelial to mesenchyme transition (EMT) (Thiery, 2002). Epithelial to mesenchyme transition

*Correspondence: Professor G Murphy;

E-mail: gm290@cam.ac.uk

Received I December 2005; revised 9 March 2006; accepted I 5 March 2006; published online I 8 April 2006 describes the de-differentiation of polarised epithelial cells to mesenchymal cells, which characteristically have a more motile phenotype. Such a loss of epithelial characteristics is often found in carcinomas with a greater malignant potential and EMT is believed to occur during the transition of adenomas to adenocarcinomas in colorectal cancer (Thiery, 2002). Extracellular matrix (ECM) degrading proteases (including serine-, metallo- and cysteine proteases) play a key role in the invasion of tumour cells, and these could function as the mediators of the EMT-induced invasiveness.

In this study, we used a somatic cell line knockout model to study the cancer phenotype induced by disrupting p300 (Iyer et al, 2004a). Loss of p300 in HCT116 cells results in a potentially aggressive phenotype characterised by reduced adhesion and increased migration. In addition, several critical genes involved in these pathways are differentially expressed, suggesting that p300 loss promotes EMT.

\section{MATERIALS AND METHODS}

\section{Cell culture, generation of $\mathrm{p}^{300^{-}}$and rescue cells}

HCT116 (ATCC, Manassas, VA, USA) and its derivatives were cultured in McCoy's 5A medium with $10 \%$ fetal calf serum (FCS) and penicillin/streptomycin (Invitrogen, Paisley, UK). p300 gene targeting was performed as previously described (Iyer et al, 2004a). HCT116 cells were chosen because they are amenable to homologous gene recombination and p300 has been found to be mutated in many colorectal cancers. Briefly, the single expressed p300 allelle in HCT116 was targeted by homologous recombination 
resulting in cells null for any expressed p300 protein. Three separately targeted clones were derived and used in each experiment. Rescue clones were made by co-transfecting pcDEFFlag-p300 (kind gift from MA Ikeda) (Suganuma et al, 2002), with pPGK-Puro at a $10: 1$ ratio, and selecting with $1 \mu \mathrm{g} \mathrm{ml}^{-1}$ Puromycin (Sigma, Cambridge, UK) for $24 \mathrm{~h}$. Two separate EF-wt rescue clones were derived and used in each experiment. p300 expression levels in each clone were determined at the start of every experiment. Rescue cells were generated using a p300 expression construct under the control of an EF-promoter (human elongation factor $1 \alpha$ promoter). This was preferred as it resulted in stable expression over longer time periods (Suganuma et al, 2002).

\section{Immunofluorescence localisation}

Cells were cultured on cover slips, fixed with $4 \%$ paraformaldehyde (PFA) solution, blocked with $1 \mathrm{~mm}$ glycine ( $\mathrm{pH}$ 7.5) and permeabilised for $4 \mathrm{~min}$ in $0.1 \%$ Triton. Immunofluorescent detection was carried out in duplicate (Roghi and Allan, 1999). The primary antibodies used were: E-cadherin (BC Biosciences, San Jose, CA, USA, 1:50), ZO-1 (Invitrogen, Zymed, Paisley, UK, $1: 100), \beta$-catenin (Transduction Labs, $1: 50$ ), Vimentin (DAKO, $1: 50)$. The AlexaFluor 488 conjugated anti-mouse antibody (Invitrogen, Molecular Probes, Paisley, UK, 1:500) was the secondary antibody in all cases. Pictures were taken with $a \times 63$ objective.

\section{Western blots}

Cells were harvested in protein lysis buffer $(50 \mathrm{~mm}$ Tris/ $\mathrm{HCl}$, $150 \mathrm{~mm} \mathrm{NaCl}, 1 \% \mathrm{NP}-40 \mathrm{v} \mathrm{v}^{-1}, 10 \mathrm{~mm}$ EDTA, Complete ${ }^{\mathrm{TM}}$ Inhibitor Cocktail 1 tablet $10 \mathrm{ml}^{-1}$ (Roche Diagnostics, Welwyn Garden City, UK)). Samples were subjected to reducing SDS-PAGE and transferred to nitrocellulose using a semidry blotter. The membranes were blocked in 5\% fat-free milk and subsequently incubated in the primary and secondary antibody solution for $1 \mathrm{~h}$ each. Blots were visualised using the Enhanced chemi-luminescence detection system from Amersham. All experiments and blots were performed in triplicate and representative examples are shown in the figures. Cytosolic and nuclear fractions were obtained with the BioVision Nuclear/Cytosol Fractionation Kit. Membrane fractionation was performed as previously described (Smith et al, 2005). Primary antibodies were mouse monoclonal E-cadherin (Zymed; 1:500), Vimentin (DAKO; 1:250), ZO-1(Zymed; 1:500), $\alpha$-tubulin (B-152; $1: 5000$ ), the polyclonal rabbit anti-p300 (Santa Cruz Biotechnology, Santa Cruz, CA, USA, $1: 200)$ and anti- $\beta$-actin (ab8227; $1: 5000)$. The secondary antibody was a sheep anti-mouse or a donkey anti-rabbit horseradish peroxidase conjugated monoclonal antibody (Jackson Immuno Research Europe, Soham, UK, $1: 5000)$.

\section{Matrigel growth}

For three-dimensional (3-D) cultures, cells were plated at a density of $1 \times 10^{5} \mathrm{~cm}^{-2}$ onto reconstituted (pre-gelled) basement membrane (Matrigel; BD Biosciences, San Jose, CA, USA) in DMEM/F12 media with $2 \%$ FCS. Cells were incubated for $18 \mathrm{~h}$ and photographed live by phase microscopy. For quantification of cluster sizes, cells were subsequently fixed in $4 \%$ PFA in PBS for $10 \mathrm{~min}$ and nuclei stained with $0.5 \mu \mathrm{g} \mu \mathrm{l}^{-1}$ DAPI for $5 \mathrm{~min}$. Cells were mounted, and cluster sizes were determined by counting nuclei using a Zeiss Axiphot microscope equipped for epifluorescence. For immunoflurescence, 3-D basement membrane cultures of cells were maintained on glass coverslips and grown to subconfluence. Cells were fixed with methanol for $15 \mathrm{~min}$, rinsed three times with PBS and blocked in PBS with $1 \%$ bovine serum albumin (BSA).

\section{Determination of integrin cell surface levels}

Cell surface levels of integrins were quantified by flow cytometry. Cells, $2.5 \times 10^{5}$, were detached using $5 \mathrm{~mm}$ EDTA in PBS, centrifuged at $200 \times \mathbf{g}$ for $4 \mathrm{~min}$ and washed in ice-cold PBS three times. All reagents used from this stage on were precooled on ice. Cells were incubated in $100 \mu \mathrm{l}$ PBS containing $0.5 \%$ BSA and the primary antibody at $10 \mu \mathrm{g} \mathrm{ml}^{-1}$ for $1 \mathrm{~h}$ on ice (integrin $\alpha_{2}$ was purchased from Dianova, integrin $\alpha_{1}$ from Serotec, integrin $\alpha_{5}$ from DAKO, integrin $\alpha_{6}$ from BD Pharmingen, integrin $\alpha_{\mathrm{V}}$ from Chemicon, integrin $\beta_{1}$ from $\mathrm{BD}$ Pharmingen, integrin $\beta_{3}$ from Serotec). Cells were washed in PBS/BSA and incubated in $100 \mu \mathrm{l}$ PBS/BSA solution containing the secondary antibody (AlexaFluor 488 goat antimouse, AlexaFluor 488 goat anti-rat $1: 500$ in the PBS/BSA solution). The cells were fixed in $2 \%$ PFA for $20 \mathrm{~min}$ and washed once in PBS. Experiments were conducted in duplicate.

\section{Adhesion assay}

Black immunotreated 96-well plates (Nunc maxisorb) were coated overnight with the matrix components in coating buffer at $10 \mu \mathrm{g} \mathrm{ml}^{-1}$ and blocked for $2 \mathrm{~h}$ with $100 \mu \mathrm{l}$ coating buffer $/ 1 \%$ BSA (Sigma Aldrich, Gillingham, UK) solution. As negative control wells were blocked with the BSA solution only. Cells were removed from their culture vessels with $5 \mathrm{~mm}$ EDTA in PBS and were dye loaded with $2 \mu \mathrm{M}$ CMFDA cell tracker dye (Molecular Probes) according to the manufacturer's protocol. $10^{5}$ cells were incubated in HBSS with $1 \mathrm{~mm} \mathrm{Mn}^{2+}$ at $37^{\circ} \mathrm{C}$ for $1.5 \mathrm{~h}$ in the coated wells. All samples were analysed in quadruplicate. After incubation the fluorescence was measured in a plate reader (Tecan Spectrafluor Plus, excitation $485 \mathrm{~nm}$, emission $595 \mathrm{~nm}$ ). The wells were washed $5 \times$ with $200 \mu$ l HBSS. The fluorescent values of each sample were averaged and the mean background value was subtracted. To inhibit the adhesion mediated by integrin $\alpha_{2}$ an integrin $\alpha_{2}$ binding antibody (Dianova, A.1.43) was added during the adhesion phase of the assay. The antibody was used at concentrations between $1 \mu \mathrm{g} \mathrm{ml}^{-1}$ and $30 \mu \mathrm{g} \mathrm{ml}^{-1}$ for the inhibition of adhesion.

\section{Boyden chamber migration assay}

The relative migration of wild-type (WT) and $\mathrm{p} 300^{-}$cells was studied with an in vitro migration assay, conducted in Boyden chambers (Becton Dickinson, $8 \mu \mathrm{m}$ pores). The membrane of the cell culture insert was coated with $100 \mu \mathrm{l}$ collagen I $\left(100 \mu \mathrm{g} \mathrm{ml}^{-1}\right.$, Sigma) or growth factor-reduced matrigel $\left(200 \mu \mathrm{lml}^{-1}, \mathrm{BD}\right.$ Biosciences, San Jose, CA, USA) in coating buffer $\left(0.1 \mathrm{M} \mathrm{NaHCO}_{3}\right.$ (pH 9.8)) for $16 \mathrm{~h}$. WT and $\mathrm{p} 300^{-}$HCT116 cells were dye loaded with $2 \mu \mathrm{M}$ CMFDA cell tracker dye (Molecular Probes). Cells, $2.5 \times 10^{5}$, were added to the cell culture insert and incubated for $22 \mathrm{~h}$. Membranes were then fixed for $5 \mathrm{~min}$ in methanol at $-20^{\circ} \mathrm{C}$, mounted on a slide and 15 randomly chosen fields of view were photographed at $40 \times$ magnification. Cells were counted, averaged over the three membranes and expressed in relation to the WT.

\section{cDNA Synthesis and qPCR}

RNA was isolated with the Promega kit according to the manufacturers protocol. In all, $1 \mu \mathrm{g}$ of total RNA was reverse transcribed using $2 \mu \mathrm{g}$ random hexamers (Amersham Biosciences, Amersham, UK) and 200 Units of Superscript II reverse transcriptase (Invitrogen Life Technologies, Paisley, UK), according to the supplier's instructions. Quantitative polymerase chain reaction (PCR) reactions were performed on a ABI Prism 7700 (Applied Biosystems, Warrington, UK; (Nuttall et al, 2003), with each reaction containing $5 \mathrm{ng}$ of reverse-transcribed RNA. Primers and fluorogenic probes for a selection of matrix metalloproteinase (MMPs) and a disintegrin and metalloproteinase (ADAMs) were 
obtained by courtesy of D Edwards, UEA, Norwich (Koshy et al, 2002; Nuttall et al, 2003).

\section{RESULTS}

Comparison of HCT116 and $\mathrm{p} 30^{-}$cells reveals an expression profile associated with EMT and a disruption in cell-cell adhesion

HCT116 derivatives null for p300 (p300- cells) were generated as previously described (Iyer et al, 2004a), and three independently targeted $\mathrm{p} 300^{-}$clones $\left(\mathrm{p} 300^{-} 1, \mathrm{p} 300^{-} 2, \mathrm{p} 300^{-} 3\right)$ were used for all subsequent experiments. p300 expression was tested by Western blot (Figure 1). To characterise the effect of p300 loss on gene expression, cDNA microarray profiling was initially performed using the $6 \mathrm{~K}$ genomewide cDNA microarrays (from ICR, Sutton, UK) as previously described (Ahmed et al, 2004). Data from these experiments showed that $\mathrm{p} 300^{-}$cells have a gene expression profile characteristic of EMT. These include a reduced expression of several members of the cadherin and integrin families and CD44. Downregulation of E-cadherin and upregulation of Vimentin was observed in two of the three clones, but overall neither were statistically significant (G Iyer and C Caldas, unpublished).

We therefore carried out an analysis of key adhesion molecules using more reliable techniques. Immunostaining showed that Ecadherin levels at adherens junctions were significantly lower in p300- cells (Figure 2A). Reverse transcription-polymerase chain reaction (RT-PCR) analyses (data not shown) and Western blot (Figure $3 \mathrm{~A}$ ) confirmed reduced transcription of the E-cadherin gene and reduced protein levels. $\beta$-Catenin and $\mathrm{ZO}-1$ were found to be redistributed in $\mathrm{p} 300^{-}$cells (Figure 2A): decreased staining of $\beta$-catenin and ZO-1 at cell-cell junctions and increased nuclear $\beta$-catenin staining (Figure $2 \mathrm{~B}$ ). The increase in nuclear $\beta$-catenin levels was verified by cellular fractionation and Western blot (Figure 4A), while no change in cytosolic $\beta$-catenin was observed (Figure 4B). Densitometry of three separate experiments showed that the increase in nuclear $\beta$-catenin was statistically significant ( $P$-value $<0.05$, Figure $4 \mathrm{~B})$. Membrane fractionation confirmed the reduced levels of $\mathrm{ZO}-1$ localising to the membrane in the p300 cells in comparison to the WT cells (Figure 4C). Total cellular levels of both proteins, determined by Western blots, remained unchanged (data not shown). Immunostaining (Figure 2A) and Western blots (Figure 3B) for the mesenchymal intermediate filament Vimentin, showed that all three $\mathrm{p} 300^{-}$clones expressed significant amounts of the mesenchymal marker Vimentin (albeit with varying degrees of heterogeneity), while levels were undetectable in HCT116 cells. These results suggest that loss of p300

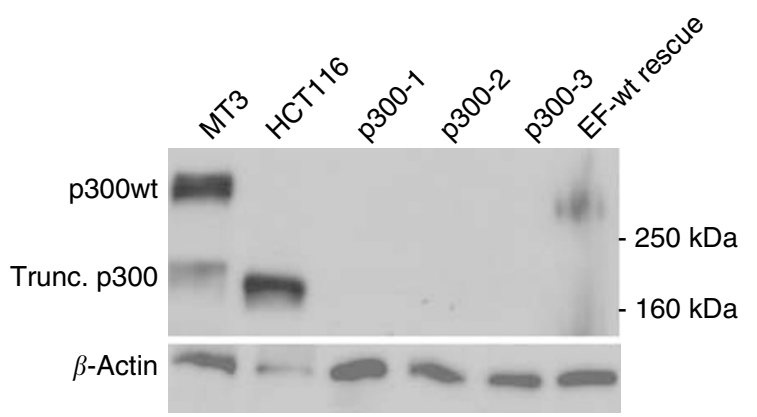

Figure I Western blot of p300. Immunoblots showing p300 levels in HCT I I 6 WT, three independently targeted p300- clones (p300- I, p3002, p300-3) and two EF-wt rescue clones, where p300 was reintroduced, with $\beta$-actin loading control. MT3 cells express the full-length p300 construct that was introduced in the EF-wt rescue clones, whereas HCTII 6 express an truncated form. The experiment was repeated three times and a representative blot is displayed. is associated with changes characteristic of EMT in this colorectal cancer cell line.

Loss of cell-cell junctions coincides with reduced clustering in matrigel

To identify abnormalities in cell - cell adhesion, cells were grown in reconstituted basement membrane (matrigel) based 3-D cultures. Under these conditions, $\mathrm{p} 300^{-}$cells grew in small, loosely adherent clusters with ragged edges characteristic of cells with defects in cell-cell adhesion compared to parental HCT116 (Figure 5A).

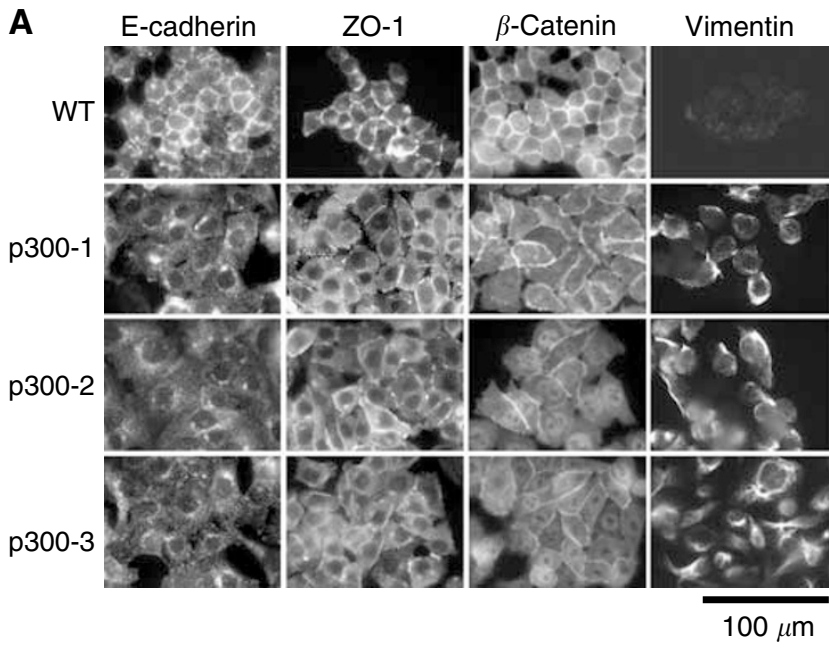

B

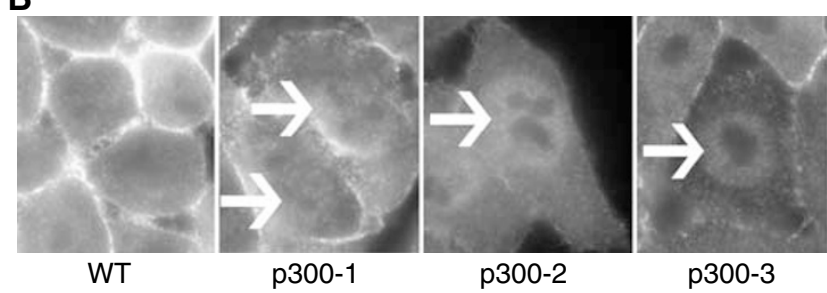

Figure 2 Immunofluorescent localisation of epithelial and mesenchymal markers. Cells were stained for the epithelial markers E-cadherin, ZO-I, $\beta$ catenin and the mesenchymal marker Vimentin $(\mathbf{A})$. Experiments were repeated three times; one representative field of view is represented for each cell type. (B) Shows a magnified version of the nuclear staining of $\beta$ catenin in the WT compared to the $300^{-}$cells.

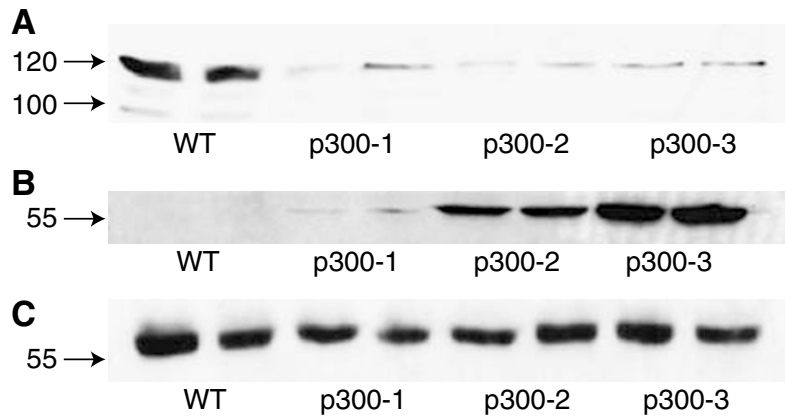

Figure 3 Western blots of $(\mathbf{A})$ E-cadherin and (B) Vimentin. Total cel lysates were analysed in duplicate from independent wells. Monoclonal mouse primary antibodies and a sheep anti-mouse horseradish peroxidaseconjugated secondary antibody were used for detection. (C) shows staining $\alpha$-tubulin on the same membrane as a loading control. The experiments were repeated three times and a representative blot is displayed. 
A

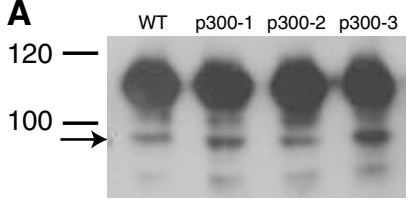

C

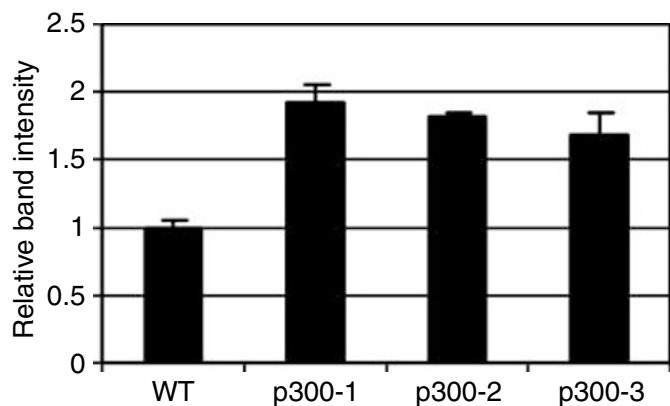

D

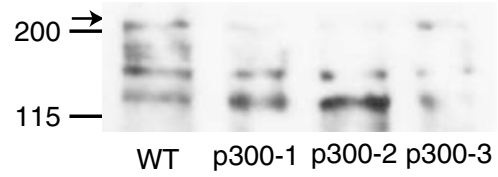

Figure 4 Western blots of $(\mathbf{A})$ nuclear, (B) cytosolic extracts, $(\mathbf{C})$ statistical analysis of $\beta$-catenin and (D) Western blot of the membrane fractionation for ZO-I. Nuclear and cytosolic extracts were prepared from WT and $\mathrm{p} 300^{-}$cells and analysed by Western blot. The $\beta$-catenin band at $92 \mathrm{kDa}$ is indicated by an arrow. The band at $110 \mathrm{kDa}$ is nonspecific and serves well as internal standard. Experiments were repeated three times and densitometry and statistical analysis of the three separate experiments (in C) showed a significant increase of $\beta$-catenin in the p300- cells ( $P$-value $<0.05$ ). (D) Shows a blot of ZO-I in membrane fractions of WT and p300- cells. The band at $225 \mathrm{kDa}$ indicated by an arrow corresponds to ZO-I. The experiment was repeated three times and a representative blot is shown.

Reintroduction of p300 in ET-wt rescue clones could reverse this phenotype. Expression of p300 in the rescue clones was confirmed by Western blot (Figure 1). Quantification of this defect confirmed that WT and ET-wt rescue clones indeed formed larger cell clusters than $\mathrm{p} 300^{-}$cells (Figure 5B). The difference between $\mathrm{p} 300^{-}$clones and WT or ET-wt rescue clones was significant for clusters up to five cells $(P$-value $<0.05)$ as well as for clusters above 10 cells $(P$-value $<0.005)$.

$\mathrm{p}^{300}{ }^{-}$cells have reduced levels of $\alpha_{2}$-integrin and reduced adhesion to collagen-I and IV

To assess whether the absence of $\mathrm{p} 300^{-}$results in abnormal cellular expression of integrins, flow cytometry analyses of surface integrins were performed. These experiments showed a decrease in $\alpha_{2}$-integrin (Figure 6), with no significant change in $\alpha_{1^{-}}, \alpha_{5^{-}}, \alpha_{V^{-}}$, $\alpha_{6^{-}}, \beta_{1^{-}}$and $\beta_{3}$-integrin levels (data not shown). Major ligands of $\alpha_{2} \beta_{1}$-integrin are type-I and IV collagen. Therefore, we tested if reduced integrin $\alpha_{2}$ levels affected cell-matrix adhesion in p300 cells. In vitro adhesion assays showed that $\mathrm{p} 300^{-}$cells adhered less to collagen I and matrigel, compared to HCT116 cells ( $P$-value $<0.05)$. In contrast, no significant difference in adhesion could be detected for fibronectin $(P$-value $>0.05)$, which is not a ligand of integrin $\alpha_{2}$ (Figure 7A). Inhibition of integrin $\alpha_{2}$-mediated adhesion by addition of an integrin $\alpha_{2}$ blocking antibody reduced adhesion of WT and $\mathrm{p} 300^{-}$cells to collagen I and matrigel significantly (for the highest antibody concentration $P<0.05$ ), however, as expected not the adhesion to fibronectin (Figure $7 \mathrm{~B}$ and $\mathrm{C})$.

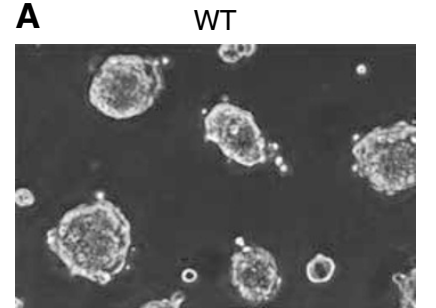

EF-wt rescue 1

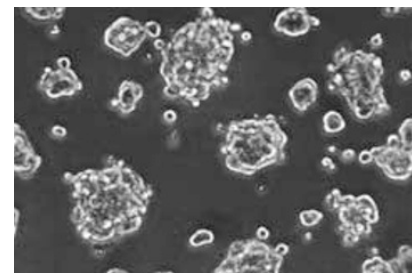

EF-wt rescue 2
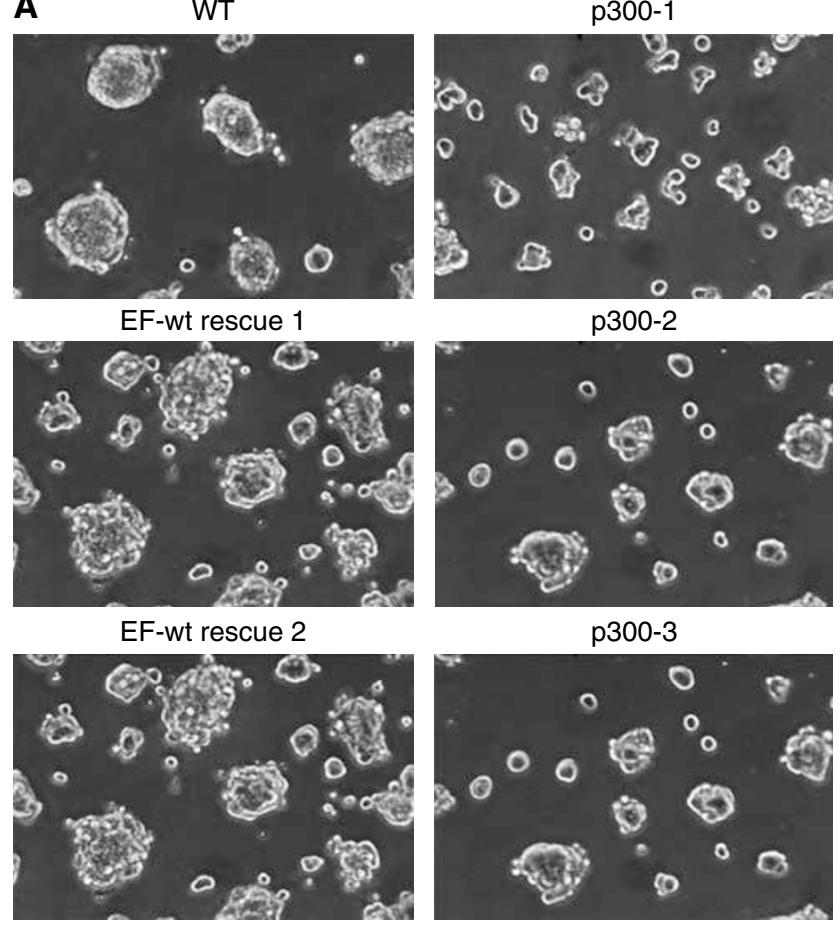

p300-2

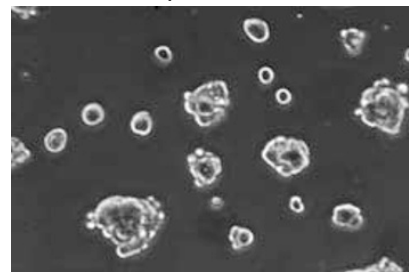

p300-3
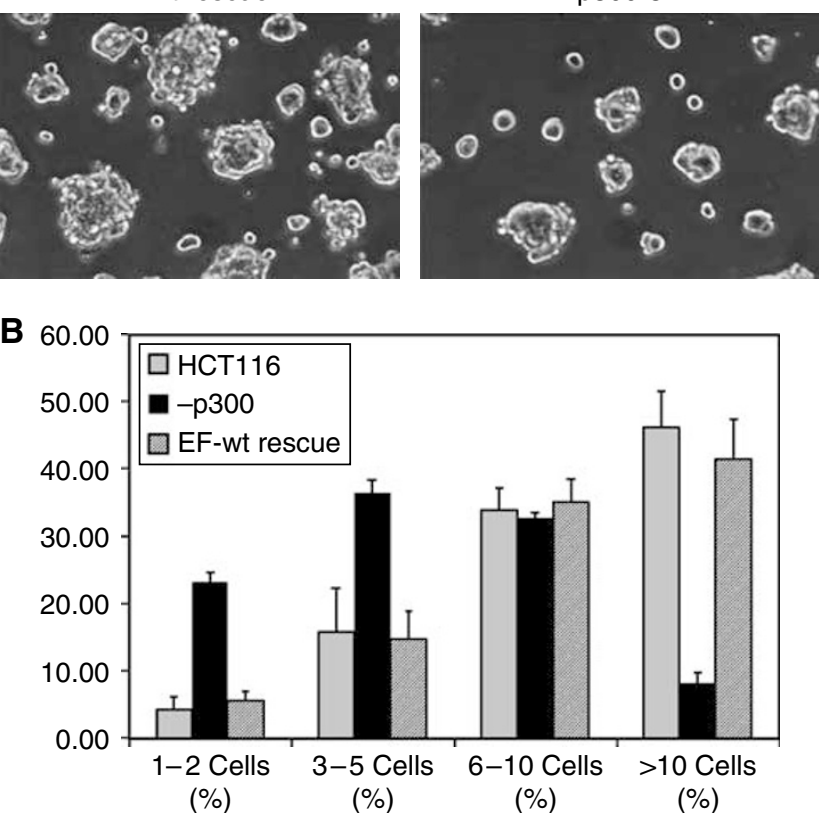

Figure 5 Adhesion defect on Matrigel growth after $18 \mathrm{~h}$. (A) Matrigel growth of WT, p300- and EF-wt rescue cell clusters. p300- clones (p300I, p300-2 and p300-3) cluster poorly into small clusters with ragged edges compared to WT cells. This defect is reversed in EF-wt rescue clones. Experiments were performed in triplicate, and the figure above shows a representative example. (B) Graph of cluster size distribution in HCTI I6, p300 and EF-wt rescue cells. The graph demonstrates the distribution of cluster sizes in $\mathrm{HCTI} 16$ vs the p300- and EF-wt rescue clones. Experiments were performed in triplicate in all three p300- clones (p300-I. p300-2 and p300-3) and two EF-wt rescue clones (EF-wt rescue I and EF-wt rescue 2). Error bars indicate one standard deviation.

These results show that HCT116 cells rely primarily on $\alpha_{2}$ integrins for adhesion to collagen I and IV and suggest that the decrease in cell surface integrin $\alpha_{2}$ levels seen in p300- cells results in reduced adhesion.

\section{p300 ${ }^{-}$cells show increased migration through collagen-I and matrigel-coated membranes}

Defects in adhesion are frequently associated with increased ECM invasion and migration in cancer cells. To compare the motility of HCT116 and $\mathrm{p} 300^{-}$cells we used a transwell assay with type-I collagen or matrigel-coated membranes. Migration through the membrane was followed for $22 \mathrm{~h}$ in response to a chemotactic 


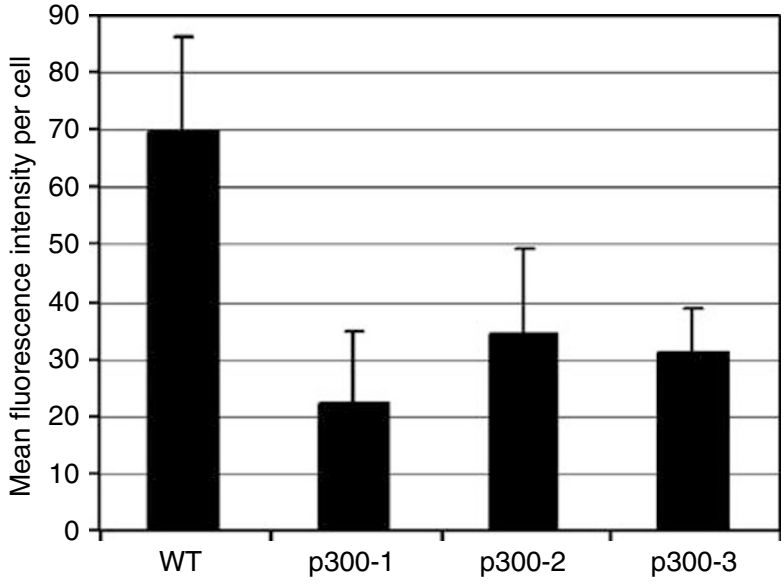

Figure 6 Comparison of integrin $\alpha_{2}$ cell surface levels in WT and $\mathrm{p}^{300^{-}}$ cells. The samples were measured in duplicate by flow cytometry. The background fluorescence of the secondary antibody was subtracted from the values. The experiment was repeated three times.
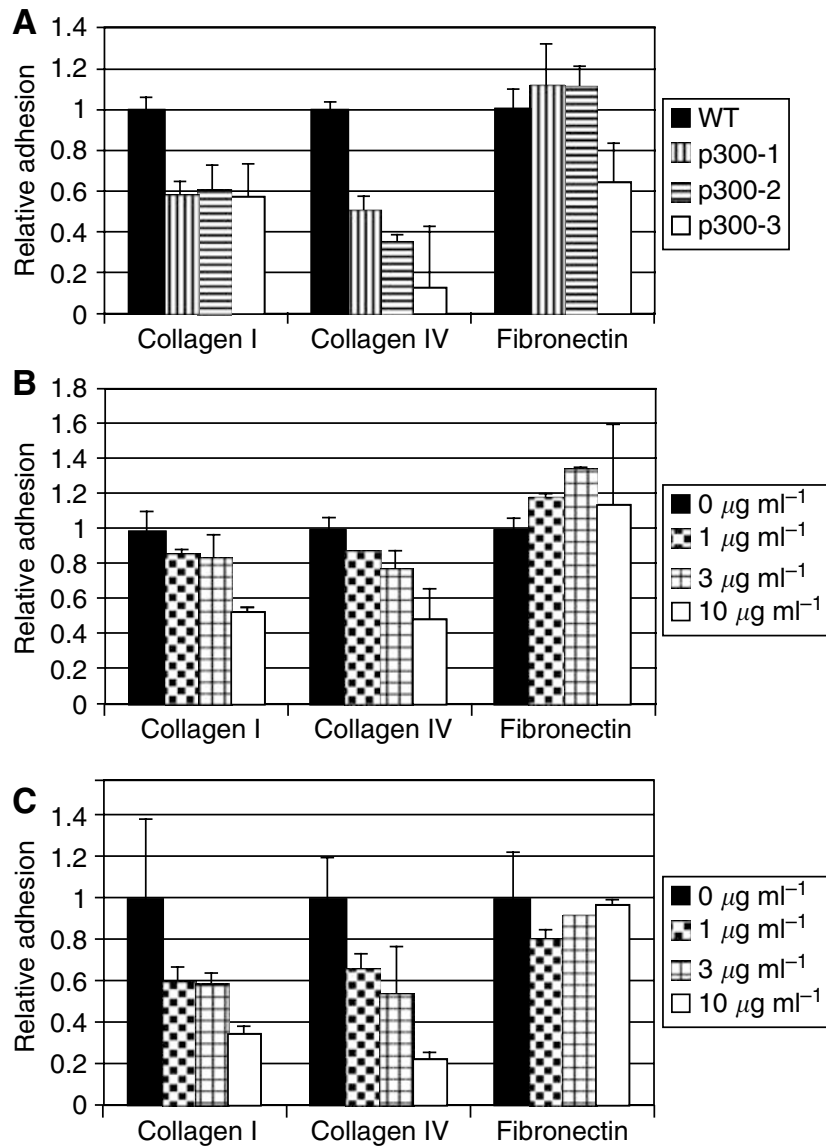

Figure 7 Adhesion of WT and $\mathrm{p} 300^{-}$cells. (A) Adhesion to different ECM components. (B and $\mathbf{C}$ ) show the inhibition of integrin $\alpha_{2}$-mediated adhesion in the WT (B) and $\mathrm{p} 300^{-}$cells (C). The concentrations in the legend indicate the concentration of the adhesion blocking integrin $\alpha_{2}$ antibody present. Samples were measured in triplicate and experiments repeated three times.

gradient of FCS. In these assays, $\mathrm{p} 300^{-}$cells demonstrated markedly increased migration through both type-I collagen and matrigel when compared to the WT HCT116 cells. Degradation of ECM and cellular invasion is believed to be primarily dependent
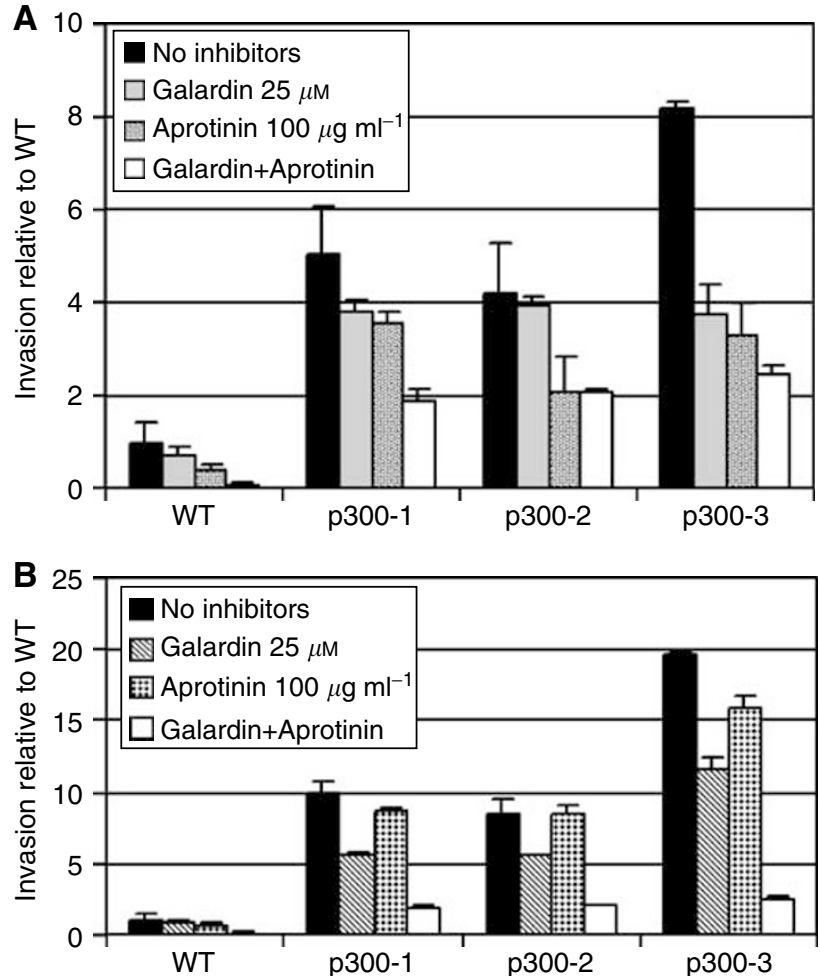

Figure 8 Migration through matrigel $(\mathbf{A})$ and collagen-I (B) coated membranes in a chemotractive FCS gradient. Experiments were conducted in triplicate and repeated three times. Where indicated metalloproteinase(Galardin at $25 \mu \mathrm{M}$ ) or the serine protease inhibitor (aprotinin at $100 \mu \mathrm{M}$ ) were added to the medium in the well and the insert.

on the proteolytic activity of metalloproteinase (MP) and serine protease activity, either by direct degradation of the ECM or as part of a protease activation cascade (DeClerck et al, 2004). We showed that migration through type-I collagen was more dependent on MP activity than serine protease activity: GM6001 or Galardin (a broad spectrum MP inhibitor) was more efficient in inhibiting migration through type-1 collagen, compared to aprotinin (a general serine proteinase inhibitor) (Figure 8A). The inhibitor solvent DMSO alone did not affect invasion (data not shown). In contrast, migration through matrigel was inhibited to a similar extent by both aprotinin and Galardin (Figure 8B). In both cases the combination of Galardin and aprotinin significantly inhibited cell migration, but could not abrogate it completely. The motility of the cells on plastic in a wounding assay was not affected by the p300 disruption (data not shown).

\section{Characterisation of MP and TIMP expression in HCT116 and $\mathrm{p} 300^{-}$cells}

Our results suggest that increased migration of $\mathrm{p} 300^{-}$cells through type-I collagen, could be MP dependent. We therefore proceeded to quantify mRNA levels of known MMP, ADAM, their inhibitors, the tissue inhibitor of metalloproteinase (TIMPs) and members of the serine protease uPA cascade, to assess whether altered expression of these proteins could account for the invasive phenotype observed. Table 1 summarises the results of the realtime RT-PCR analyses. The majority of MPs, TIMP-2 and -3, as well as components of the uPA system were unchanged. However, the mRNA levels of MMP-7, -15 and ADAM15 were significantly decreased in $\mathrm{p}^{300^{-}}$cells (Figure 9). MMP-7 and ADAM15 were found to be below the limits of detection by Western blot (data not shown). 
Table I Summary of the effect of p300 ablation on MP, serine protease and TIMP mRNA levels

\begin{tabular}{clcl}
\hline MMP & Effect of p300 ablation & ADAM & Effect of p300 ablation \\
\hline 1 & Low expression & 8 & Low expression \\
2 & Low expression & 9 & No change \\
3 & No expression & 10 & No change \\
7 & Downregulation** & 12 & No change \\
8 & No expression & 15 & Downregulation** \\
9 & No expression & 17 & No change \\
10 & No change & 19 & No change \\
11 & No change & 28 & No expression \\
12 & No change & 33 & No expression \\
13 & No change & TIMP & \\
14 & No change & 1 & No expression \\
15 & Downregulation** & 2 & No change \\
16 & No expression & 3 & No change \\
17 & No change & 4 & No expression \\
19 & No change & Plasmin system & \\
23 & No change & uPAR & No change \\
25 & No change & uPA & No change \\
& & Plasminogen & No expression \\
& & PAl- I & No change \\
& & PAl-2 & No change
\end{tabular}

MP, serine protease and TIMP mRNA levels were determined in triplicate by PPCR and repeated three times. Low expression signifies a $C_{T}$-value $<35$, where quantification becomes inaccurate. Downregulation *** corresponds to a $P$-value $<0.00$ I when a mean of the three $\mathrm{p} 300^{-}$clones is calculated vs the WT.

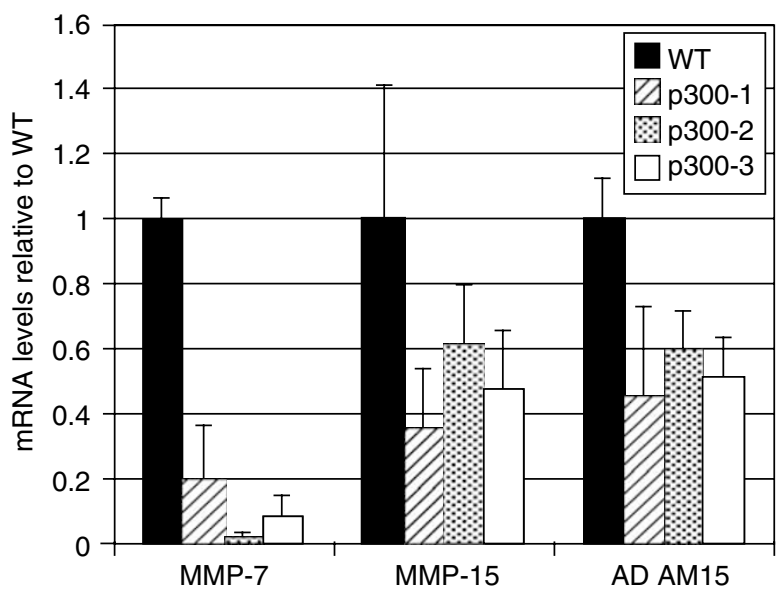

Figure 9 The effect of p300 on the mRNA levels of MMP-7, - I5 and ADAM I5. MP and TIMP mRNA levels were determined by qRCR in three separate experiments that were conducted in triplicate. The relative expression values were obtained by normalising the $c_{T}$-values using the $c_{T}$-values of the $18 \mathrm{~S}$ in the samples as reference, compensated for the exponential fluorescence increase and set in relation to the WT values. $c_{T}$-values of MPs in WT: MMP-7: 30; MMP-15: 25; ADAMI 5: 24.

\section{DISCUSSION}

p300 is considered to be a putative tumour-suppressor gene because it is mutated, deleted or underexpressed in a number of cancers. However, the mechanisms, through which p300 functions to suppress tumorigenesis, remain controversial (Chan and La Thangue, 2001; Iyer et al, 2004b). Using previously generated p300 deficient clones, derived from a colorectal carcinoma cell line, we propose a novel pathway through which p300 may function as a tumour suppressor.

Disrupting p300 in HCT116 cells induced changes characteristic of EMT, a process thought to be important in carcinogenesis. p300- cells had significant cell-cell adhesion defects, with downregulation of E-cadherin, a major component of the epithelial adherens junctions, and reduced aggregation in matrigel cultures. Reintroduction of p300 could restore the aggregation phenotype. In addition $\beta$-catenin was found to be increased in nuclear fractions of $\mathrm{p} 300^{-}$cells, which may contribute to intracellular signalling inducing EMT. In colorectal tumours, tumour cells at the invasion front display strong nuclear $\beta$-catenin, and the Tcf4$\beta$-catenin transcription factor complex is thought to be involved in EMT (Brabletz et al, 2005). p300- cells also showed reduced adhesion to the basement membrane components collagen I and IV consequent to reduced cell surface levels of integrin $\alpha_{2}$. The effect of integrin cell surface levels on the invasiveness of cancer cells is debatable and probably depends on the system that is used and relative surface levels and activation status of integrins. Sun et al (1998) reported that reexpression of the $\alpha_{2} \beta_{1}$-integrin in a poorly differentiated breast carcinoma cell line, Mm5MT, resulted in reversion of a malignant phenotype to a differentiated epithelial phenotype. Reduction of integrin $\alpha_{6} \beta_{4}$ via siRNA in MDA_MB_231 breast cancer cells resulted in decreased invasion on a laminincoated surface (Lipscomb et al, 2003).

Epithelial to mesenchyme transition is often characterised by an increase in invasive potential in vitro, and this is believed to foster the invasive and metastatic potential of tumour cells (Thiery, 2002). Comparing the migrative potential of parental HCT116 and $\mathrm{p} 300^{-}$cells in vitro, we found that $\mathrm{p} 300^{-}$cells showed increased migration through collagen I and matrigel-coated membranes. It was not possible to assess migration through a thicker layer of either gel (which would be more representative of cellular invasion), since invasion rates were too low to obtain statistically significant results. Nonetheless, we showed that migration through matrigel was serine- and metalloproteinase dependent, whereas the invasion through collagen I was metalloproteinase and only marginally serine protease dependent.

Interestingly, increased migration was found to be independent of expression levels of proteases involved in collagen or matrigel degradation. Cell surface levels of MMP-14 (determined by flow cytometry) and MMP-2 and MMP-9 activity (determined by zymography) were not affected by the p300 disruption (data not shown). Previous studies have shown that MMP-7 and MMP-14 can be upregulated as a consequence of the movement of $\beta$-catenin to the nucleus and the increase in the Tcf4- $\beta$-catenin transcription factor complex. Previous studies have shown that factors other than $\beta$-catenin can also affect the MMP-7 and MMP-14 promoter activity and the nature of such effectors in this system remain to be elucidated (Crawford et al, 2001; Takahashi et al, 2002). Other targets of this gene complex include CD44, which was also shown to be downregulated in the microarray analysis of $\mathrm{p} 300^{-}$cells. We also noted that MMP-15 was significantly downregulated in $\mathrm{p} 300^{-}$ cells. Wound healing experiments did not show increased motility of the p $300^{-}$cells on a plastic culture dish (data not shown). These data indicate that the migration in a chemotactic gradient is supported by the degradation of the ECM coat. However, since protease levels are largely unaffected by the p300 ablation, the increased migration of the $\mathrm{p} 300^{-}$cells is most likely due to decreased cell-cell interactions and cell-matrix adhesion. The disruption of cell-cell junctions may allow single $\mathrm{p} 300^{-}$cells to migrate more readily through the $8 \mu \mathrm{m}$ pores of the membrane compared to the clusters of the HCT116 WT cells, as many colon cancer cell lines migrate as cohorts rather than single cells.

The data presented here proposes a novel mechanism through which p300 could function as a tumour suppressor: p300 disruption promotes EMT and an aggressive cancer phenotype. It has been suggested that histone deacetylase (HDAC) inhibitors, which are currently being tested in clinical trials for cancer therapy, function to selectively promote the expression of tumoursuppressor genes. We propose an additional anticancer mechanism, where HDAC inhibitors could promote p300 histone acetylase 
activity, hence inhibiting EMT and increased invasiveness seen in cancer cells.

\section{ACKNOWLEDGEMENTS}

We thank MA Ikeda for providing the p300 rescue construct. We are indebted to Caroline Pennington and Dylan Edwards,
University of East Anglia, Norwich for guidance and reagents for mRNA analysis by QRT-PCR. DK was supported by the DAAD and Trinity College. NGI was the recipient of an NMRC (Singapore) Medical Research Fellowship. WRE was supported by the British Heart Foundation and GM by Cancer Research UK and the European Union framework 5 programme (QLK3-CT2002-021360 and framework 6 programme (LSHC-CT-2003503297). CC was supported by Cancer Research UK.

\section{REFERENCES}

Ahmed AA, Vias M, Iyer NG, Caldas C, Brenton JD (2004) Microarray segmentation methods significantly influence data precision. Nucleic Acids Res 32: e50

Brabletz T, Hlubek F, Spaderna S, Schmalhofer O, Hiendlmeyer E, Jung A, Kirchner $\mathrm{T}$ (2005) Invasion and metastasis in colorectal cancer: epithelial-mesenchymal transition, mesenchymal-epithelial transition, stem cells and beta-catenin. Cells Tissues Organs 179: $56-65$

Chan HM, La Thangue NB (2001) p300/CBP proteins: HATs for transcriptional bridges and scaffolds. J Cell Biol 144: 2363-2373

Crawford HC, Fingleton B, Gustavson MD, Kurpios N, Wagenaar RA, Hassell JA, Matrisian LM (2001) The PEA3 subfamily of Ets transcription factors synergizes with beta-catenin-LEF-1 to activate matrilysin transcription in intestinal tumors. Mol Cell Biol 21: 1370-1383

DeClerck YA, Mercurio AM, Stack MS, Chapman HA, Zutter MM, Muschel RJ, Raz A, Matrisian LM, Sloane BF, Noel A, Hendrix MJ, Coussens L, Padarathsingh $M$ (2004) Proteases, extracellular matrix, and cancer: a workshop of the path B study section. Am J Pathol 164: 1131-1139

Gayther SA, Batley SJ, Linger L, Bannister A, Thorpe K, Chin SF, Daigo Y, Russell P, Wilson A, Sowter HM, Delhanty JD, Ponder BA, Kouzarides T, Caldas C (2000) Mutations truncating the EP300 acetylase in human cancers. Nat Genet 24: $300-303$

Goodman RH, Smolik S (2000) CBP/p300 in cell growth, transformation, and development. Genes Dev 14: $1553-1557$

Iyer NG, Chin SF, özdag H, Daigo Y, Hu DE, Cariati M, Brindle K, Aparicio S, Caldas C (2004a) p300 regulates p53-dependent apoptosis after DNA damage in colorectal cancer cells by modulation of PUMA/p21 levels. Proc Natl Acad Sci USA 101: 7386-7391

Iyer NG, özdag H, Caldas C (2004b) p300/CBP and cancer. Oncogene 23: $4225-4231$

Koshy PJ, Lundy CJ, Rowan AD, Porter S, Edwards DR, Hogan A, Clark IM, Cawston TE (2002) The modulation of matrix metalloproteinase and ADAM gene expression in human chondrocytes by interleukin-1 and oncostatin M: a time-course study using real-time quantitative reverse transcription-polymerase chain reaction. Arthr Rheum 46: 961 -967
Lipscomb EA, Dugan AS, Rabinovitz I, Mercurio AM (2003) Use of RNA interference to inhibit integrin (alpha6beta4)-mediated invasion and migration of breast carcinoma cells. Clin Exp Metast 20: $569-576$

Muraoka M, Konishi M, Kikuchi-Yanoshita R, Tanaka K, Shitara N, Chong JM, Iwama T, Miyaki M (1996) p300 gene alterations in colorectal and gastric carcinomas. Oncogene 12: $1565-1569$

Nuttall RK, Pennington CJ, Taplin J, Wheal A, Yong VW, Forsyth PA, Edwards DR (2003) Elevated membrane-type matrix metalloproteinases in gliomas revealed by profiling proteases and inhibitors in human cancer cells. Mol Cancer Res 1: 333-345

Ozdag H, Batley SJ, Forsti A, Iyer NG, Daigo Y, Boutell J, Arends MJ, Ponder BA, Kouzarides T, Caldas C (2002) Mutation analysis of CBP and PCAF reveals rare inactivating mutations in cancer cell lines but not in primary tumours. Br J Cancer 87: $1162-1165$

Roghi C, Allan VJ (1999) Dynamic association of cytoplasmic dynein heavy chain 1a with the Golgi apparatus and intermediate compartment. J Cell Sci 112(Part 24): 4673-4685

Smith JM, Dornish M, Wood EJ (2005) Involvement of protein kinase C in chitosan glutamate-mediated tight junction disruption. Biomaterials 26: $3269-3276$

Suganuma T, Kawabata M, Ohshima T, Ikeda MA (2002) Growth suppression of human carcinoma cells by reintroduction of the p300 coactivator. Proc Natl Acad Sci USA 99: 13073-13078

Sun H, Santoro SA, Zutter MM (1998) Downstream events in mammary gland morphogenesis mediated by re-expression of the a2 $\beta 1$ integrin: the role of the a6 and $\beta 4$ integrin subunits. Cancer Res 58: 22242233

Takahashi M, Tsunoda T, Seiki M, Nakamura Y, Furukawa Y (2002) Identification of membrane-type matrix metalloproteinase-1 as a target of the beta-catenin/Tcf4 complex in human colorectal cancers. Oncogene 21: $5861-5867$

Thiery JP (2002) Epithelial-mesenchymal transitions in tumour progression. Nat Rev Cancer 2: $442-445$ 